\title{
Protée
}

\section{La fin du sexuel. Expérience, expérimentation et pratiques sexuelles dans les fictions de Dennis Cooper}

\section{Jean-Ernest Joos}

Volume 27, numéro 3, 1999

L’imaginaire de la fin

URI : https://id.erudit.org/iderudit/030574ar

DOI : https://doi.org/10.7202/030574ar

Aller au sommaire du numéro

Éditeur(s)

Département des arts et lettres - Université du Québec à Chicoutimi

ISSN

0300-3523 (imprimé)

1708-2307 (numérique)

Découvrir la revue

Citer cet article

Joos, J.-E. (1999). La fin du sexuel. Expérience, expérimentation et pratiques sexuelles dans les fictions de Dennis Cooper. Protée, 27(3), 93-102.

https://doi.org/10.7202/030574ar
Résumé de l'article

Quelle est la fin du sexuel ? C'est la question que l'on pose ici dans la convergence des travaux de la psychanalyse sur les pulsions de mort, des éléments d'une culture de la mort qui s'approprie la mort comme lieu de fantasmes sexuels, et les fictions de Dennis Cooper, auteur américain contemporain qui réfléchit sur les relations entre les limites du sexuel et celles de la vie. L'hypothèse est que dans un monde posthumain, où l'unité de l'expérience de la mort et du sexuel s'est perdue, les imaginaires générés par la négation de la finitude sont en fait des lieux d'expérimentation des limites du sujet et des formes nouvelles de communauté. 


\section{LA FIN DU SEXU EL EXPÉRIEN CE, EXPÉRIM ENTATION ET PRATIQ UES SEXU ELLES DANS LES FICTIONS DE DEN NIS COOPER \\ JEAN-ERNEST JOOS}

Les récits et discours de la fin peuvent être analysés en fonction de leur contenu imaginaire, en tant que mythe, ou en tant que délire. Mais ils peuvent aussi être entendus dans leur relation aux sujets qui les produisent, les transmettent et s'y reconnaissent individuellement et collectivement. Ils apparaissent alors comme des lieux de fuite ou d'expérimentation des limites du sujet. Les imaginaires de la fin ont, du point de vue du sujet, tous en commun la négation de la finitude humaine: l'homme, ou ce qu'il en reste, croit avoir survécu à la fin du monde, il croit avoir l'omniscience de sa fin, ou alors il a perdu le sens de sa propre mort au point de ne plus y reconnaître la vérité de sa finitude. Il s'agira alors de faire ressortir dans l'imaginaire de la fin, comme négation de la finitude, toute la dimension exploratoire qui relève de la redéfinition de l'homme. Pour ce faire, nous avons choisi un auteur américain contemporain.

Cet auteur, Dennis Cooper, a fait son entrée sur la scène littéraire au tout début des années quatre-vingt et figure régulièrement dans les travaux universitaires, en raison de la complexité de ses constructions narratives et de la profondeur de ses réflexions sur le lien entre le sexe et la mort. Nous l'avons choisi, pour notre part, non pas à cause de l'héritage littéraire qu'il perpétue, celui de Bataille notamment, mais parce qu'il n'a jamais cessé de faire partie d'un circuit populaire et marginal de lecteurs fascinés par la pornographie violente, souvent de mauvais goût, les meurtriers, surtout les «serial killers», les adolescents drogués et prostitués, et par d'autres éléments encore marqués par la perte du sens de la différence entre la vie et la mort, la réalité et la fiction, le bien et le mal.

L'homosexualité qui teinte les situations et les personnages ne fait paradoxalement que renforcer cet ancrage des romans de Cooper dans la culture de la mort, dans la mesure où le lectorat gay (y compris celui de romans érotiques) qui s'est constitué lors des mouvements de revendication identitaire des années quatre-vingt semble difficilement s'y reconnaître. C'est donc à partir d'une lecture de quelques-unes des fictions de Cooper que nous proposerons une interprétation de l'imaginaire de la fin, entendu comme perte du sens de la finitude dans un univers posthumain. 
ÊTRE EN RELATION AVEC LA MORT...

Le sexe et la mort. On pourrait se contenter de cela, montrer comment Dennis Cooper reprend et travaille ce couple qui s'est élaboré depuis au moins le début du siècle dans les écrits de Freud et de Bataille. Mais ce serait se nier comme lecteur, comme récepteur, se défaire trop rapidement de ces expériences limites qui non seulement font l'objet d'une description, mais sollicitent de plus la participation active du lecteur consentant. Ainsi, Cooper utilise des références à des genres populaires tels que le film d'horreur et la pornographie afin de jouer sur les habitudes et les attentes d'un public déjà structuré. D'autres affects, du dégoût à l'attraction, sont convoqués dans cette actualisation d'une performativité textuelle. Il s'agira donc de mettre celleci en évidence, afin de montrer quelle relation singulière avec le lecteur les textes tentent d'établir. On entend ici par relation l'un des objets privilégiés aussi bien de la psychanalyse que de l'anthropologie. Une relation est ce qui permet au même de rencontrer, de reconnaître et de symboliser son Autre. C'est sur ce point que les fictions de Cooper trouvent leur originalité et leur "contemporanéité», dans la mise en place d'une relation tout à fait singulière autour de la sexualité et de la mort, qui permet une redéfinition du singulier, du collectif et de l'universel.

La sexualité et la mort sont en effet des lieux de reconnaissance identitaire possible. Au moment où Cooper écrit ses fictions, l'idée d'une sexualité gay, d'une culture et d'une littérature gay fait déjà partie des conditions de circulation des textes. C'est dans cet espace identitaire que Cooper introduit l'altérité, le non-reconnaissable, notamment la fin du sexuel. De même, en faisant de la mort un lieu d'appropriation imaginaire et d'expérimentation illimitée, il fait éclater l'unité de l'expérience de la mort et rejoint ainsi la réflexion qui s'est faite sur la communauté et la mort, de Heidegger à Blanchot et à Lyotard. Le sexe et la mort: leur lien devient une question de partage ou de non-partage d'une expérience commune, ou impossible. L'imaginaire de la mort et le déni de la finitude engendrent chez Dennis Cooper des formes originales de relation qui viennent élargir la compréhension de la sexualité.

\section{LA QUESTION DE L'EXPÉRIENCE HUMAINE ET LES LIMITES DE L'EXPÉRIMENTATION}

L'expérience n'est pas l'expérimentation. Cette dernière, lorsqu'elle est totale, s'oriente vers le sanslimite. L'expérience au contraire suppose toujours la finitude. Elle se révèle même comme limite et pose la question d'un sujet. L'expérimentation ne relève que de l'action, de la structure de l'action et des conditions exigées d'un agent pour actualiser un projet. Dans les deux fictions de Dennis Cooper, Closer et Frisk que l'on propose ici à l'analyse, les deux dimensions sont clairement présentes.

L'expérimentation totale, qu'il s'agisse de soi ou de l'autre, est privilégiée. Elle se manifeste par une réduction du corps à une chose sur laquelle va s'accomplir le projet d'une abolition de toute limite, notamment entre la vie et la mort, entre l'image et la réalité, le sexuel et le non-sexuel. Ainsi, certains personnages «objectifient» leur corps au point de se regarder en train d'être manipulés par d'autres, au point de désirer leur propre mort dans un rapport sexuel. D'autres n'éprouvent de désir que s'ils peuvent fantasmer la mort de leur partenaire sexuel, ou sont amenés à érotiser la mort et, par-delà la mort, l'intérieur du «corps assassiné».

Pourtant, cette expérimentation totale qui refuse que la mort soit la limite du sexuel, qui cherche tout à la fois à réduire le corps à une chose et à érotiser cette matière inerte, n'est aucunement transparente à ellemême. Autrement dit, la fin de l'expérimentation n'est pas dans le fait d'expérimenter. Sa dimension première est plutôt esthétique et cognitive. D'une part, la mort est belle, elle est le moment de pure adéquation du corps à son image. D'autre part, on cherche à savoir ce qui fait l'érogénéité du corps, à trouver où se cache le secret du corps sexué. L'expérimentation vise l'au-delà de la sexualité, elle cherche à saisir ce moment où le sexuel cesserait d'être sexuel, là où le sexuel toucherait à sa fin. Pour saisir le sens de l'expérimentation totale, il faut donc 
comprendre quelle relation est présupposée entre le sujet et son Autre (le cadavre, la mort, la fin du sexuel, etc.). C'est à ce moment que l'on doit inscrire l'expérimentation dans l'idée d'expérience, en posant la question: de quoi l'expérimentation est-elle l'expérience?

L'expérimentation illimitée de soi que l'on retrouve dans les fictions de Cooper inscrit cet auteur dans un courant aisément identifiable dans la littérature américaine contemporaine ${ }^{1}$. Ce courant se reconnaît à un questionnement sur le devenir-marchandise du corps, sur son devenir-chose, ce que les Anglo-saxons nomment, en utilisant une terminologie marxiste, "commodification of the body». Autour de cette objectivation du corps gravite un certain nombre de thématiques: la fascination pour l'idée et l'image du "serial killer», le tueur qui n'entretient pas d'autre rapport singulier à son crime que celui de son propre système et de ses propres fantasmes, la mort comme accomplissement ultime et absolu de l'acte sexuel, la volonté de connaître le corps jusque dans son intérieur et la sexualisation de cette pulsion cognitive comme volonté de puissance et esthétisation de la mort, la confusion totale entre l'image du corps et le corps érogène lui-même (une confusion générée par les médias et la pornographie) et, par conséquent, l'abolition de la différence entre le corps vivant et le cadavre, et même la croyance que la rigidité de la mort est l'état où se révèle l'image du corps.

Toute cette culture et tout cet imaginaire construits autour de l'objectivation du corps traversent aussi bien la littérature que le cinéma, notamment dans les films dits de série $\mathrm{B}$, films d'horreur à petit budget et à contenu «soft porn». L'inscription de Dennis Cooper dans cette culture marque la coupure de celui-ci avec l'héritage de Bataille. Là où le travail de Bataille sur le rapport entre le sexe et la mort reste motivé par une théorie anthropologique de la transgression et de l'excès, l'écriture de Cooper participe plutôt d'un monde où rien ne semble pouvoir être plus violent et transgressif que le devenir-chose du corps. L'expérimentation ne vise pas le dépassement des limites, mais le sans-limite dans un monde qui a déjà perdu le sens de la limitation. Voici quelques exemples puisés parmi une infinité d'éléments illustrant le processus de "commodification of the body». On y reconnaîtra les deux dimensions dominantes du rapport au corps objectivé, la dimension esthétique et la dimension cognitive:

I've got this longstanding urge to really open up someone I'm hot for. (Frisk, p. 53)

I want to know every thing about you. But, to really do that, I'd

have to kill you. (Frisk, p. 67)

Then we cut him apart for a few hours, and studied everything inside the body. (Frisk, p. 106)

He looked so beautiful with his eyes empty. (Frisk, p. 93)

Do you want to know what's inside that cute body of yours?»

(Closer, p. 99)

[...] being dead, he is perfect. (Closer, p. 129)

Thank you for being so well designed, kid. (Closer, p. 37) ${ }^{2}$

Face à l'état des choses signifié par ces citations, peut-on se contenter de constater cette perte du sens de la mort et des frontières du sexuel? Il y a quelque chose de tautologique à lire cette thématique comme un symptôme de l'époque - époque postmoderne du capitalisme avancé. L'expérimentation illimitée du sexuel serait le signe de la déshumanisation et de l'assujettissement du corps au système d'échange. Cela n'est certes pas faux, mais quels seraient alors le désir et la fin que porte la compulsion à expérimenter? N'est-ce pas constituer un cercle vicieux que d'y voir le signe de la déshumanisation et de l'aliénation du corps, le signe de ce qu'elle viserait en même temps à accomplir? À rester prisonnier de ce cercle, on perd le sens de l'écriture, de la possibilité d'une interrogation sur le pouvoir de l'homme à donner un sens à ses actions. La répétition fictionnelle de la compulsion ne vise pas simplement à la perpétuer. On s'en rend compte simplement par les affects de lecture multiples que Dennis Cooper provoque. À la fascination esthétique de la mort en image répond encore tout un ensemble de réactions à caractère moral - répulsion, dégoût -, qui rappellent toutes au corps le sens de la limite. Il n'y a aucune raison de postuler un lecteur aussi froid ou indifférent que la plupart des acteurs 
des scènes décrites. L'expérimentation même totale n'abolit pas la question de l'expérience des limites.

La deuxième dimension, la dimension de l'expérience, bien que moins explicite que celle de l'expérimentation, est pourtant tout à fait élaborée dans l'écriture de Cooper. L'expérience est toujours expérience de la finitude, c'est-à-dire des limites, non pas de celles que l'on rencontre en face de soi, mais de celles qui nous déterminent et qui se font jour à la faveur de nos tentatives d'autodépassement. On reprend ici le concept d'expérience tel qu'on le retrouve dans la relecture que Heidegger et Benjamin ont faite de la philosophie kantienne et postkantienne. L'expérience englobe l'ensemble des conditions de possibilité du «rapport au monde» en tant que relation finie. Or, avec Dennis Cooper, tous les sujets d'expérimentation découvrent à un moment ou à un autre une finitude qui leur rappelle que leur coappartenance au monde leur assigne les limites indépassables du possible. Le désir d'érotiser la mort, tout particulièrement de faire coïncider le rapport sexuel et le moment de la mort, se solde toujours par un échec. Lorsque la mort est là le sexe n'y est plus, et lorsque l'érotisme est présent la mort n'est plus représentable.

Cette non-coïncidence n'est pas donnée, elle est révélée au sujet comme résultat de son expérimentation. Et ce résultat n'est plus désormais "objectivable», puisque la limite n'est plus limite de l'objet se retirant, mais limite du sujet même dans ses capacités de représentation. Le "serial killer» présumé de Frisk, après avoir massacré un adolescent afin de l'analyser, conclut le compte rendu épistolaire de son meurtre ainsi: «When I try to picture him, I go blind and my cock gets unbelievably hard» (p. 107); «Quand je m'efforce de l'imaginer, je perds la vue et mon sexe devient incroyablement rigide». Toutes les images pornographiques de la mort se révèlent, en dernière instance, n'être que des images. Ainsi, Frisk s'ouvre et se ferme sur une double description d'un corps mutilé qui apparaît réel au premier abord, mais qui s'avère à la toute fin n'être qu'une mauvaise mise en scène du corps maquillé d'un acteur pornographique. La mort reste bien infigurable, sans visage.
Pourtant, ce caractère infigurable n'empêche pas de produire de la fiction. Bien au contraire. L'imaginaire de la mort s'articule de façon indissociable à son infigurabilité, composant une expérience globale, mais fragmentaire, du corps vivant. Les voix narratives et la construction de Closer illustrent cette fragmentation de façon quasi didactique, à un point tel que l'on croirait lire une mise en image de la théorie que Lyotard propose du postmoderne. Dans son texte "Réponse à la question: qu'est-ce que le postmoderne?», celui-ci caractérise en effet le postmoderne par la fin de l'«unité de l'expérience» (1988:15-16), et dans le Différend, il précise cette thèse en montrant que les trois champs identifiés par Kant dans ses Critiques, à savoir les champs cognitif, éthique et esthétique, ne peuvent plus prétendre trouver leur point d'unité. Or, dans Closer, on est clairement en présence, dans l'expérience de la mort, de ces trois dimensions et, à chaque fois, le fragment d'expérience rencontre sa limite dans l'impossibilité de faire la synthèse des deux autres. L'esthétisation de la mort est omniprésente, mais, malgré la détermination des sujets, elle ne produit aucunement une proximité de la mort même, elle ne fait que renforcer la valeur érotique des images en laissant la mort dans l'infigurable.

L'expérience cognitive, quant à elle, apparaît à peu près à tous les stades de l'expérimentation limite de soi et de l'autre. Tuer, ouvrir le corps, voir l'intérieur, toutes ces actions constituent des actes et des fantasmes qui sont identifiés comme des tentatives de savoir ce qui fait l'érogénéité du corps, ce qui différencie le corps d'une chose. L'échec de ce désir de savoir est montré de multiples façons. Georges ne connaît le moment où sa mère meurt que par un signe sur le moniteur (Closer, p. 96). Tom, l'adepte de «snuff movies», se voit obligé d'indiquer aux spectateurs le moment exact où la victime est morte ("Now»), tant l'instant de la mort est imperceptible à l'écran (Closer, p. 109). Et le dépeçage des corps ne renvoie jamais à autre chose qu'à l'érotisation de l'acte, de l'acte de tuer et de mutiler, jamais à une réponse du corps qui viendrait livrer le secret de sa matérialité. 
Quant à la dimension éthique, elle apparaît dans un personnage central, Philippe qui, bien que porteur lui aussi du désir de tuer et de baiser un cadavre, se rend compte à la fois qu'il en est moralement incapable et que c'est cette incapacité même qui est à la source de son fantasme (Closer, p. 110-111). Mais, pas plus qu'une autre dimension, l'éthique - «tu ne tueras point" - n'a ici le dernier mot de l'expérience de la mort, car elle n'englobe pas la dimension esthétique à laquelle elle continue de renvoyer: "Death is beautiful».

Pour comprendre véritablement le travail de Cooper sur le sexe et la mort, il faut donc le situer dans le cadre de cette expérience globale fragmentée à laquelle le lecteur est soumis. Et Cooper, à la suite de Closer, va chercher à mettre en place des formes narratives complexes afin d'explorer des formes nouvelles de performativité.

Ainsi, aux voix narratives collectives de Closer, répond la structure dialectique de Frisk, construit autour d'une fiction à l'intérieur du récit. Les mises en scène de violences les plus extrêmes sont exposées dans une lettre dont ni le destinataire, ni le lecteur ne savent au moment de la lecture si elles sont réelles ou fictives. Et lorsque l'on découvre après coup qu'elles ne sont que des fantasmes, il est trop tard pour annuler l'ensemble des affects (attraction, horreur, fascination, etc.) que la lecture a produits. Ce dispositif narratif poursuit un double objectif: montrer, d'une part, que le fictionnement de la mort contient en lui-même un moment où réalité et imaginaire sont indissociables et, d'autre part, lier ensemble, dans une même expérience globale, la mise en image de la mort et son caractère infigurable, en faire les deux faces dialectiquement interdépendantes, mais inconciliables, d'un même rapport sexualisé à la mort.

\section{PULSIONS DE MORT ET SEXUALITÉS SINGULIĖRES}

Quelle est la fin du sexuel? À quel moment cesse-ton d'être sexué? Avec la mort qui se dérobe, c'est toute l'unité de l'expérience sexuelle qui se perd. C'est en ce sens que les romans de Dennis Cooper sont "posthumains", puisque l'évidence d'une nature humaine y fait défaut. On ne saurait dire «ici, avec la mort, le sexuel trouve sa fin». L'infigurabilité de la mort laisse intact le désir d'érotiser sa propre mort et celle de l'autre. Elle signifie que la mort ne peut plus servir de référence ultime, de moment de vérité du soi $^{3}$.

Lire ou comprendre Dennis Cooper revient à saisir le travail fait sur l'éclatement de l'expérience sexuelle. L'auteur joue de façon tout à fait explicite sur certaines des conditions socioculturelles de la lecture, faisant appel à la complicité du lecteur et misant en même temps sur sa possible exclusion. Pratiques sexuelles et pratiques de lecture se répondent donc.

Ainsi, les images de cadavres érotisés renvoient à une culture populaire, celle des films d'horreur à caractère pornographique, qui fonctionnent sur un mode purement performatif: un scénario minimal qui sert de prétexte à des scènes de meurtres, de mutilation et d'exposition de cadavres dans des poses sexuelles. Cette culture populaire présuppose un public déjà «éduqué» qui sait faire bon usage de ce matériel.

De même, Cooper fait appel à une culture pornographique, à laquelle il emprunte un mode précis de mise en scène des corps, plus particulièrement des actes sexuels. Il s'agit de la mise en scène frontale, de l'exposition la plus explicite possible, sans ombre, sans nuance et sans métaphore. À ces références à la culture pornographique, populaire et commerciale, s'ajoute une visée vers un groupe spécifique de lecteurs. À la différence de ce qui se passe dans les films d'horreur, où il n'y a de victimes que féminines, les romans de Cooper sont situés dans un monde presque exclusivement homosexuel. Ils contiennent des références à des pratiques sexuelles courantes dans ce monde - par exemple le «rimming»-, mais difficiles à identifier pour un public non initié, et dont la définition ne figure pas dans un dictionnaire usuel ${ }^{4}$. Mais cette complicité avec un public lecteur homosexuel, Cooper joue à la compromettre, en introduisant dans son texte des éléments repoussants qui seraient absolument prohibés dans une production de pornographie gay. Nous songeons notamment à ces 
acteurs de scènes érotiques qui apparaissent étonnés, indifférents, et parfois même dégoûtés par les gestes sexuels posés à leur égard, et qui viennent ainsi briser le consensus sexuel essentiel à toute bonne pornographie. Cooper mêle constamment une altérité du dehors et une altérité du dedans. La première forme est celle de la non-reconnaissance liée à des identités divergentes: ignorance ou répulsion devant une sexualité qui n'est pas la nôtre. La seconde forme est celle d'une altérité qui menace de l'intérieur un espace de reconnaissance commun, elle relève du nonreconnaissable. Bien entendu, c'est celle-là surtout qui intéresse Cooper, la première forme étant là pour mettre en scène la seconde. Ainsi, l'auteur crée des espaces d'intimité - par exemple, un univers gay - pour les menacer ensuite de violences symboliques. La sexualité est soumise au même questionnement que la mort, toutes deux rendues étrangères au soi.

Par cette double altérité, Cooper introduit dans la compréhension de la sexualité un concept nouveau qui vient rompre l'opposition simple entre l'universel et le singulier: le concept de la relation. L'altérité dans sa double forme se problématise toujours au sein de relations singulières. L'expérience dont il est question est une expérience relationnelle. Elle porte sur les possibilités et les limites de la relation à l'altérité, à la mort, au corps et au sexuel.

La scène originelle des fictions de Cooper le montre clairement. Être tué dans un rapport sexuel et le meurtre comme acte sexuel ultime sont deux modes de relations sexuelles à la mort. Et ces relations sexuelles sont non seulement fantasmées, mais aussi mises en ouvre et en acte, de telle sorte que leurs possibilités et limites sont explorées. Peut-on entrer en relation avec son propre corps mort, peut-on érotiser un cadavre? Et dans un univers posthumain où l'évidence des limites s'est perdue, rien n'indique $a$ priori que de telles relations ne soient pas possibles.

Cooper multiplie les situations de non-réciprocité où des relations hétérogènes se croisent. Le meilleur et le plus fréquent cas de non-réciprocité est l'expérience de l'abject liée à la sexualité anale. Ce qui pour l'un est un objet d'intense érotisation sera pour l'autre un objet de pure répulsion, ou de curiosité pour les tiers. Les trois moments d'une telle scène sont représentés dans les chapitres centraux de Closer: il y a Georges, l'objet passif et consentant, son "utilisateur", Philippe, qui désire absolument faire un bon usage de la beauté de celui-ci (p.50-51), enfin un spectateur qui tentera de reproduire sur le même sujet passif, Georges, la scène à laquelle il a assisté (p. 67). L'objectif de ces mises en scène, comme de ces autres où les adolescents rejouent des scènes de films pornographiques, est de montrer que les trois positions - la position de celui qui contrôle et désire, la position de celui qui est utilisé et la position de ceux qui regardent - ne trouvent jamais leur point d'entente ${ }^{5}$.

La mort, de même, devient la médiation problématique de relations non réciproques. L'un demande à être tué, l'autre ne demande qu'à tuer avec le consentement de sa victime, et pourtant les deux demandes ne se rencontrent jamais. C'est qu'avec Dennis Cooper le propre d'une relation n'est pas dans sa réciprocité, mais dans le simple fait qu'il y a de la relation et non pas la négation de toute relation.

Dans la réflexion sur le sexe et la mort, Cooper introduit un troisième terme, un signifiant qui désigne l'horizon de cet univers posthumain: «numb», «numbness». Le signifiant renvoie à la perte de toute sensation; le corps ou l'esprit se figent, se glacent, s'engourdissent, etc. Dans l'usage que Cooper fait du signifiant, il marque la fin du sexuel, le déplacement du sens de la mort. Un chapitre complet de Frisk a ce terme comme titre, celui-là même où apparaît le compte rendu des carnages extrêmes. Ce chapitre suit et répond à un passage qui figure au début du livre. Ces deux moments constituent le chiasme qui structure tout l'ouvrage. Car si les meurtres relatés vers la fin du récit s'avéreront purement imaginaires, la tentative de meurtre qui les annonce est, par contre, une histoire bien réelle. Mais selon la logique même de la narration, le désir de meurtre ne sera pas mené à terme.

One night I got Samson so loaded he walked like the carpet was quicksand or something. He couldn't speak, I don't think. I 
aimed him at the bed, where he fell. I knelt over his chest and gazed down at his face until it blurred. Then I punched it.

Again. I sort of lost my way, I can't remember exactly. Things

were breaking. Sometimes I'd catch one of Samson's eyes

studying me, which I guess was a muscle reflex.

I should include some reaction shots here, I know, but I doubt I

had many. I felt numb, blank... (Frisk, p. 34)6

La perte de sensation est décrite dans Closer, de façon identique, comme entretenant un lien intime avec le désir de mort, vu cette fois-ci du point de vue de celui qui désire être tué.

"Kill me», the silhouette rasped. "I can't feel anything. I mean you're okay. Shit. I don't know... I guess I wanted somebody to kill me for over a year or whatever so don't fucking worry".

(Closer, p. 12) 7

C'est pour échapper à l'état décrit dans cet extrait que les sujets cherchent leur propre mort.

C'est également la menace permanente de la perte du sexuel qui amène la fascination pour l'érotisation de la mort, que cette fascination soit portée par des acteurs du texte ou par le lecteur. Tout plutôt que l'absence de sensation qui, comme on le verra à l'instant, ne signifie rien de moins que le renfermement sur soi, que l'incapacité de se lier au dehors, à l'Autre.

Par ce déplacement de l'horizon de la finitude, de la mort comme perte de la vie à la mort comme perte de son propre corps érogène, Cooper rejoint la réflexion de la psychanalyse sur la pulsion de mort.

Car il faut ici être bien clair: la pulsion de mort n'est pas dans la volonté d'appropriation sexuelle de la mort. Tuer, vouloir mourir et toutes formes de pulsion de destruction appartiennent encore aux pulsions de vie. La pulsion de mort est bien plutôt dans la possibilité du retrait du corps hors de l'érogénéité ("I can't feel anything»). Et c'est très exactement ce que Freud proposait. Il faut situer Cooper dans la lignée des lecteurs de Freud qui ont choisi de lier la pulsion de mort à une théorie générale de la sexualité, elle-même construite autour d'une compréhension relationnelle de la pulsion de mort. On songe ici à des auteurs tels que Michel de M’Uzan (1977) et François Péraldi (1984).

On considère aussi le texte déterminant de Freud, intitulé «Problèmes économiques du masochisme». La particularité de ce texte de 1924 est qu'il utilise la notion de pulsion de mort pour comprendre une forme singulière de sexualité, le masochisme, plutôt que de l'utiliser dans le cadre d'une théorie de l'identité et du narcissisme.

La définition que propose ce texte de la pulsion de mort reste la même que dans les textes précédents de Freud, à savoir: «[...] la tendance à faire passer la vie perpétuellement changeante à la stabilité de l'état inorganique» (1973:288). La pulsion de mort n'est pas le désir de mourir, bien au contraire, elle est la tendance à abolir la différence entre la vie et la mort.

On retrouve dans ce questionnement freudien la même prise en compte de la chosification du corps que chez Cooper. La pulsion de mort fait en effet du corps vivant une matière inorganique. "To be numb" est bien un état de stabilisation, d'absence de tension par manque de désir et de sensation. La définition freudienne souligne aussi ce qui caractérise les pulsions de vie: la vie naît de la possibilité du changement, à savoir de la présence d'une différence qui amène l'instabilité. La sexualité se constitue alors sur un «alliage» des pulsions de vie et des pulsions de mort. Ce n'est pas tant la théorie freudienne du masochisme qui nous intéresse ici que le mode par lequel Freud analyse la sexualité. Il se dégage en effet de cette analyse une certaine conception de la sexualité comme réalité singulière. Chaque sexualité singulière se définit par la façon dont elle surmonte les pulsions de mort, dont elle allie pulsions de vie et pulsions de mort. Il devient possible, par hypothèse, d'identifier ce moment où la différence se marque, où l'altérité cesse d'être ce qui abolit le vivant pour devenir ce qui au contraire l'ouvre vers l'«extérieur", selon le terme de Freud (1973:291). Ainsi, dans le masochisme, selon Freud, le désir de subir la souffrance est en fait une pulsion de vie, car il dérive d'une pulsion de destruction, à l'origine tournée vers l'«extérieur», puis retournée vers soi. L'avantage 
théorique de l'hypothèse freudienne dans l'interprétation de Cooper est qu'elle évite d'isoler les fantasmes de violence de l'ensemble des relations dans lesquelles ils s'inscrivent, et qu'elle permet donc de situer les fantasmes ayant trait au fait de donner ou de recevoir la mort à l'intérieur d'une sexualité singulière, comme un élément dans une relation globale à soi et à l'autre.

Que nous proposent donc les fictions de Cooper comme modes de dépassement de la pulsion de mort? Comment échapper à la mort de son corps érogène («numbness»)?

C'est précisément le désir de tuer et d'être tué qui est porteur de la pulsion de vie. Il faut tout d'abord rappeler comment la mort intervient toujours ici dans une situation relationnelle. Le désir d'être tué est bien toujours une demande adressée à l'Autre ${ }^{8}$ - je dirais même, sans ironie aucune, une demande d'amour. De même, le désir de tuer est toujours à la recherche d'une reconnaissance.

Les meurtriers de Cooper cherchent paradoxalement des victimes consentantes ("Do you want me to kill you or not?» (Closer, p. 100)

Le dispositif narratif de Frisk met en scène cette demande de reconnaissance de façon explicite dans des lettres en rdemande de destinataires. En fait, Cooper multiplie les relations qui ont la mort comme médiation, où c'est la mort elle-même qui met en relation à l'Autre. La mort est donc bien ici du côté de la pulsion de vie. Le désir de mort est même souvent la seule forme d'espoir, la seule possibilité d'ouverture. Pourtant, ces relations médiatisées par la mort sont paradoxales. Si elles s'actualisaient selon leur finalité constitutive, elles s'aboliraient comme relation. Le moment de pure coïncidence entre le sexe et la mort, entre l'acte sexuel et la mise à mort, impliquerait que le corps est devenu une simple chose, que la chair et l'image sont confondues, que toute altérité, toute tension vers le dehors sont abolies. L'adolescent masochiste se refermerait sur sa propre inertie («numbness»), et le meurtrier atteindrait lui aussi le point d'insensibilité totale («numbness»), où son acte ne signifierait rien de plus que de tuer ce qui est déjà, et toujours déjà, mort, sa victime n'offrant plus aucune résistance vitale. Or, il se trouve que la finalité de la relation de mort est toujours mise en échec, la coïncidence du sexe et de la mort n'advenant jamais. Cette non-coïncidence est tout aussi importante dans le déroulement de l'histoire que dans ce qui est donné à lire et à vivre chez le lecteur. Le lecteur assiste en effet à la mise en place de relations médiatisées par la mort, puis à l'impossibilité de leur actualisation. Et, pourtant, malgré ces échecs, les relations en question restent bien médiatisées par la mort, mais selon un mode qui fait toute la valeur et l'originalité des fictions de Cooper. C'est cette expérience relationnelle qu'il s'agit maintenant de comprendre, car il y là une prise de position et je dirai même une solution proposée face à ce qui constitue, pour Cooper et sa génération, le problème de l'époque, celui du devenir-chose du corps ("commodification of the body»).

\section{LE DÉNI DE LA FINITUDE}

\section{ET LA MORT COMME MÉDIATION}

Identifions à nouveau les deux temps de l'expérience du corps et de la sexualité que nous avons proposés: le premier temps est celui d'un déni de la finitude, le second est le rappel de la finitude, le retournement du déni ${ }^{9}$. Il n'y a pas de troisième temps: on en reste à l'oscillation entre les deux. Le déni de la finitude se reconnaît dans l'appropriation imaginaire de la mort. Issu d'une perte de conscience («numbness») des frontières de la vie, le déni de la finitude prend la forme de fantasmes sur la mort, d'une érogénéisation du cadavre, de la croyance que la mort a un visage, qu'elle peut être mise en image. Le déni de la finitude est ainsi le ressort de la fiction. En son absence, il ne serait pas possible de faire de la fiction sur la mort. Il renforce le devenir-chose du corps et place la mort comme médiation incontournable de toute relation. Il est porteur de la pulsion de mort, puisque l'imaginaire de la mort implique la dissociation que Cooper décrit si bien dans l'expérimentation de soi et dans la sensation de l'absence de sensation («numbness»). Mais, en même 
temps, le déni de la finitude et tout l'imaginaire qui en provient sont des signes de la vie, dans la mesure où ils contiennent en eux-mêmes la possibilité de leur propre retournement. Le déni est une négation qui peut toujours se retourner, au moment où il se révèle comme déni. Ce moment se fait jour lorsque l'imaginaire de la mort se trahit comme imaginaire, lorsque l'infigurabilité de la mort rappelle à l'imaginaire ses limites. La mise en fiction de la mort ne peut être autre chose qu'une mise en fiction, l'image de la mort n'est qu'une image. Le rappel de la finitude ne fonctionne pourtant pas comme un retour du refoulé: il fait ressortir plutôt ce qui, dans l'imaginaire, appartient à la vie, à savoir la mise en relation. L'imaginaire de la mort, le déni de la finitude produisent de la relation, de l'ouverture à l'Autre. L'infigurabilité de la mort rappelle au sujet du déni qu'il y a de l'Autre dans toute relation. Là est la réponse de Cooper à la menace que subit le corps à l'époque contemporaine. Le devenir-marchandise de celui-ci, l'omniprésence de la mort contiennent en eux-mêmes un potentiel de survie. Le corps, en devenant chose, reste en relation. Son utilisation présuppose malgré tout un rapport à l'autre. Il suffit alors de faire ressortir cette possibilité relationnelle, en faisant du désir de la mort une demande à l'autre/ Autre. Il en est de même pour la mort. À une époque où le virus du VIH a imposé la possibilité de la mort au sein de toute relation sexuelle, la vision de Cooper apparaît comme étonnamment salutaire ${ }^{10}$. Elle cherche à transformer la mort en une médiation créatrice de relation, et non une médiation destructrice de relation. L'usage qui est fait ici du fantasme de l'acte sexuel et de la mise à mort sert précisément à cette création. L'imaginaire de la fin n'est pas seulement un lieu de déni du symbolique, mais aussi de symbolisation, de mise en relation ${ }^{11}$.

Ainsi, les adolescents de Closer, notamment le masochiste Georges, retrouvent l'érogénéité de leur corps, leur capacité de désirer, à la toute fin de leur expérience de l'oubli de la mort. Maintenant qu'ils ont fait l'expérience de l'Autre à travers la mort, ils peuvent resymboliser et resexualiser les parties de leur corps symboliquement et réellement mutilées. Et dans Frisk, Cooper reprend un motif déjà utilisé dans Wrong et qui puise dans la littérature du passé. Il s'agit du fantôme. Dans Wrong, le fantôme du jeune gay assassiné reste parmi les vivants, non pas pour les hanter, mais au contraire pour garder le lien. Et dans Frisk, c'est un adolescent qui, au cours d'un rêve éveillé, fait revivre un jeune gay assassiné à qui il parle de son amour pour Dennis, le présumé «serial killer», un de ces hommes qui désirent la mort de tous leurs objets de désir. Mais, au-delà de ces situations précises, tout au long des récits, lorsque les fantasmes et les récits se dissolvent, la mort, infigurable mais toujours figurée, reste comme lien privilégié entre tous les personnages, et aussi comme relation au lecteur. Ce qu'un lecteur partage avec le récit et avec tous les autres lecteurs, c'est ce même besoin de la mort comme lieu d'imaginaire partagé. La mort est notre lien. Dans La communauté inavouable, Blanchot proposait l'idée d' «une communauté négative», selon des mots repris à Bataille (1983:45), où ce qui était partagé, c'était l'incomplétude de l'existence de chacun. L'impossibilité de mettre la mort en commun est le moment de la reconnaissance commune. La nuance, la nouveauté dans la littérature postmoderne de Cooper est que la mort intervient comme troisième terme de la relation. Elle met en relation, et s'il y a de la communauté, ce n'est que par le réseau des relations plurielles qui se constituent autour d'elle. Lorsque je désire la mort, j'ouvre une possibilité qui ne s'actualisera comme relation que si la mort, par son infigurabilité, me renvoie à autre chose, à savoir à la relation sexuelle. L'enjeu est désormais dans la création de médiations efficaces. C'est là aussi la position politique de Cooper face aux identités collectives, en l'occurrence l'identité homosexuelle. Tous les personnages ont beau être gay, la relation homosexuelle ne leur est pas donnée d'emblée. La relation reste ouverte et problématique, toujours menacée par une altérité radicale (la pulsion de mort, aurait dit Freud), et la convocation de la mort, à travers les imaginaires de meurtre, est ce qui vient restaurer et rendre possible la relation. Les 
fictions de Cooper brisent les lieux de reconnaissance, que ce soient ceux de l'identité sexuelle ou ceux de l'humain comme finitude universelle, pour ensuite réintroduire et le sexe et la mort comme jonction des relations plurielles. La relation de lecture est alors ellemême toujours disjonctive et oscillante. Là où on se reconnaît, on est menacé de rejet et de répulsion, et là où on ne se reconnaît plus, on est appelé à se joindre.

\section{N O TES}

1. Voir J. Annesley, 1996.

2. "J'ai depuis longtemps cette envie d'“ ouvrir" véritablement quelqu'un qui m'excite".

"Je veux tout savoir de toi. Mais, pour y arriver, je devrais te tuer".

«Nous l'avons dépecé quelques heures durant, et nous avons fait un examen exhaustif de l'intérieur de son corps".

"Il était si beau avec ses yeux vides».

"Veux-tu savoir ce qu'il y a à l'intérieur de ton joli corps».

«[...] dans la mort, il est parfait".

"Merci d'être si parfaitement calibré, le jeune». Je traduis.

3. Il y aurait ici un rapprochement intéressant à faire entre Cooper et E. A. Poe dans la mesure où on trouve chez ce dernier un questionnement qui prend sa source dans le constat d'une délocalisation de la mort. La mort n'étant plus localisable dans l'espace et le temps, les sujets doivent se constituer dans l'absence de ce point de référence (les morts sont-ils vraiment morts?; sommes-nous déjà dans la tombe?; qui est mort et qui est vivant?; etc.). La différence entre Poe et la littérature contemporaine est cependant dans l'expérience radicale d'un déni total de la finitude.

4. Le Harrap's Shorter French and English Dictionnary (éd. 1993) nous donne au verbe " rim»: «janter (une roue); (of hill, etc.) border, encercler (une vallée); nails rimmed with dirt, des ongles ourlés de crasses ". Aucune référence n'est faite aux pratiques sexuelles oralesanales auxquelles renvoient les termes " to rim", « rimmed " et «rimming".

5. E. Jakson, Jr. (1994) propose à ce sujet des remarques intéressantes, bien que son analyse souffre de la limitation de ses présupposés qui sont ceux d'une théorie psychanalytique axée sur le sujet (frontières du sujet, relation entre le sujet et son dehors, sujet de désir, etc.). Il me semble au contraire que les problématiques de Cooper exigent plutôt une théorie générale de la sexualité à partir des possibilités données au sujet à l'intérieur de ses relations. Il va sans dire qu'une telle théorie reste encore à faire, mais que la lecture de Dennis Cooper l'annonce. 6. «Une nuit, j'ai tellement bourré Samson que le tapis sous ses pas était comme du sable mouvant, ou quelque chose de ce genre. Il ne pouvait pas parler, je ne le pense pas. Je l'ai orienté vers le lit, où il est tombé. Je me suis agenouillé au-dessus de sa poitrine, et j'ai fixé son visage jusqu'à ce qu'il devienne flou. Puis, je l'ai frappé à coups de poing. Encore. J'étais égaré, je ne me souviens pas bien. Les choses se brisaient. Par moment, j'apercevais un des yeux de Samson en train de me regarder faire, un réflexe musculaire, probablement.

À ce point-ci, je devrais inclure quelques vues de mes réactions, je le sais bien, mais je doute d'en avoir eu beaucoup. Je me sentais inerte, vide...». C'est moi qui souligne.

7. " "Tue-moi", demande la silhouette d'une voix rauque. "Je ne ressens rien. Je veux dire, t'es correct. Merde. Je ne sais pas... Je pense que cela fait plus d'un an que je cherche quelqu'un pour me tuer, ou quelque chose comme ça. Alors, fuck, t'inquiète pas" ".

8. On met une majuscule à l'Autre pour rappeler dans l'esprit de Lacan qu'il ne s'agit pas ici de quelqu'un, un autre, mais d'un autre possible, de la possibilité de l'autre.

9. On utilise le terme de déni au sens psychanalytique d'une négation qui présuppose dans ses conditions de production une affirmation occultée.

10. E. Young dans son essai interroge brièvement la tâche de la littérature (1992).

11. D. Cooper fait jouer ainsi au niveau de la culture et de la société ce ressort que Michel de M'Uzan a identifié sur le plan psychologique et individuel dans le «travail de trépas». De M’Uzan a montré comment dans le travail de trépas le déni de la mort permet au mourant d'intensifier ses relations au monde extérieur et à ses proches. De même, le déni de la mort permet à une culture posthumaine qui a perdu le sens de la finitude de réinventer ses relations avec les autres et d'augmenter les possibilités d'interactions symboliques.

\section{RÉFÉREN CES BIBLIO G RAPHIQ U ES}

ANNESLEY, J. [1996]: "Commodification, Violence and the Body: a Reading of Some Recent American Fictions", dans American Bodies. Cultural Histories of the Physique (sous la dir. de T. Armstrong), Sheffield, Sheffield Academic Press.

BlanchOt, M. [1983]: La Communauté inavouable, Paris, Minuit.

COOPER, D. [1989]: Closer, New York, Grove Weidenfeld;

[1991]: Frisk, New York, Grove Weidenfeld;

[1992]: Wrong, New York, Grove, Weidenfeld.

FREUD, S. [1973]: Névrose, psychose et perversion, Paris, P.U.F.

HeIdegGer, M. [1962]: Chemins qui ne mènent nulle part, Paris, Gallimard.

JAKSON, E. Jr. [1994] : «Death Drives Across Pornotopia: Dennis Copper on the Extremities of Being", GLQ: A Journal of Lesbian and Gay Studies, 1-2, 143-161.

LYOTARD, J.-F. [1983]: Le Différend, Paris, Minuit;

[1988]: Le Postmoderne expliqué aux enfants, Paris, Galilée.

M'UzAN, M. de [1977]: De l'art à la mort. Itinéraire psychanalytique, Paris, Gallimard.

PÉRALDI, F. [1984]: «Voyage dans l'entre-deux mort», Frayages, no 1, 19 38.

YounG, E. et G. CAVENEY [1992]: Shopping in Space: Essays on America's Blank Generation Fiction, London \& New York, Serpent's Tail. 